Revue internationale P.M.E.

Économie et gestion de la petite et moyenne entreprise

\title{
Les structures participatives d'influence du personnel sur les décisions : enquête auprès de PME
}

\section{Daniel Nebenhaus Lamsade}

Volume 3, numéro 1, 1990

URI : https://id.erudit.org/iderudit/1007948ar

DOI : https://doi.org/10.7202/1007948ar

Aller au sommaire du numéro

Éditeur(s)

Presses de l’Université du Québec

ISSN

0776-5436 (imprimé)

1918-9699 (numérique)

Découvrir la revue

\section{Citer cet article}

Nebenhaus Lamsade, D. (1990). Les structures participatives d'influence du personnel sur les décisions : enquête auprès de PME. Revue internationale P.M.E., 3(1), 89-104. https://doi.org/10.7202/1007948ar

\section{Résumé de l'article}

L'école du management participatif étend son influence sur les entreprises, et notamment au sein des PME.

Deux éléments fondent ce renouveau :

- l'intérêt croissant porté à la fonction GRH (Gestion des Ressources Humaines) dans les organisations;

- l'émergence d'une famille de techniques de gestion innovatrices reposant sur l'appel à la responsabilité collective et à l'initiative des acteurs.

Le «on fait mieux ensemble, que chacun séparément» exprime l'idée que le tout peut être supérieur à la somme des parties dans le domaine de l'action.

Cet article présente une enquête auprès de PME françaises, ayant comme problématique et comme enjeu l'état des modalités de gestion et de régulation interne de la prise de décision. Elle repose sur la connaissance du fonctionnement réel des structures et dispositifs participatifs.

Le choix des PME repose sur le constat suivant : les méthodes managériales y sont différentes de celles des grandes entreprises et par conséquent les PME constituent un champ d'investigation en soi.

Trois parties structurent l'exposé des résultats de cette recherche.

Il discute de la filiation théorique du management participatif considéré comme une école de la gestion des ressources humaines axée sur les processus de décision dans l'entreprise. Les références à des travaux de management et de sociologie des organisations permettent de dégager une perspective stratégique pour la participation.

Partant de là, l'article présente la construction des hypothèses de cette recherche, l'enquête elle- même et la méthodologie de collecte de l'information. Enfin, la troisième partie expose les variétés de dispositifs participatifs qui se sont dégagés de l'échantillon des entreprises. Deux points sont abordés : leur description, et les éléments caractéristiques de leur fonctionnement.
Ce document est protégé par la loi sur le droit d'auteur. L'utilisation des services d’Érudit (y compris la reproduction) est assujettie à sa politique d'utilisation que vous pouvez consulter en ligne.

https://apropos.erudit.org/fr/usagers/politique-dutilisation/ 


\title{
Les structures participatives d'influence du personnel sur les décisions : enquête auprès de PME
}

Daniel NEBENHAUS

LAMSADE, Université

Paris-Dauphine

\begin{abstract}
RÉSUMÉ
L'école du management participatif étend son influence sur les entreprises, et notamment au sein des PME.

Deux éléments fondent ce renouveau :

- l'intérêt croissant porté à la fonction GRH (Gestion des Ressources Humaines) dans les organisations;

- l'émergence d'une famille de techniques de gestion innovatrices reposant sur l'appel à la responsabilité collective et à l'initiative des acteurs.

Le "on fait mieux ensemble, que chacun séparément" exprime l'idée que le tout peut être supérieur à la somme des parties dans le domaine de l'action.

Cet article présente une enquête auprès de PME françaises, ayant comme problématique et comme enjeu l'état des modalités de gestion et de régulation interne de la prise de décision. Elle repose sur la connaissance du fonctionnement réel des structures et dispositifs participatifs.

Le choix des PME repose sur le constat suivant : les méthodes managériales y sont différentes de celles des grandes entreprises et par conséquent les PME constituent un champ d'investigation en soi.

Trois parties structurent l'exposé des résultats de cette recherche.

II discute de la filiation théorique du management participatif considéré comme une école de la gestion des ressources humaines axée sur les processus de décision dans l'entreprise. Les références à des travaux de management et de sociologie des organisations permettent de dégager une perspective stratégique pour la participation.
\end{abstract}

- Maître de conférence de sciences de gestion à l'Université de Paris-Dauphine, diplômé de l'École des HEC (Jouy-en-Josas, France), docteur de troisième cycle de l'Université Paris-Dauphine en économie appliquée, rattaché au Laboratoire d'analyse et modélisation de systèmes pour l'aide à la décision (LAMSADE). Adresse : LAMSADE, Université Paris IX Dauphine, Place du Maréchal de Lattre de Tassigny, 75775 Paris Cedex 16, France. 
Partant de là, l'article présente la construction des hypothèses de cette recherche, l'enquête ellemême et la méthodologie de collecte de l'information.

Enfin, la troisième partie expose les variétés de dispositifs participatifs qui se sont dégagés de l'échantillon des entreprises. Deux points sont abordés : leur description, et les éléments caractéristiques de leur fonctionnement.

\section{ABSTRACT}

The participative management schooling developps its influence over organizations, and particularly over SBE.

This revival is based on two elements :

- the rising attention gived to the management of human ressources in the organization;

- the emergence of a family of innovating management practises, built on a collective responsability of actors (personal) of the firms.

The well-known "we do better together than separately" indicates the idea that the whole can do better than the sum of the parts in the field of action.

This paper presents an original investigation about french SBE. Its problematic is about the managerial forms and internal regulation in the decision-making process. It is built on the knowledge of established running of participative structures and devices.

The choice of SBE results of the following report : their management methods are different of these of large firms, and therefore, SBE represent a particular investigation field by itself.

The results of this research are presented in three parts.

The theoritical links of participative management, considered as a human ressources management set of methods, and references to works issued of authors in management and organization sociology, gives the opportunity to show its strategical perspective.

Starting from there, this paper presents the building of the hypothesis behind this work, the investigation itself, and the methodology in order to collect the informations treated.

Finally, the third part exposes the various participative devices issued from the sample. Two aspects are more developped; the description of the devices which have been met through the investigation, and their principal features.

\section{RESUMEN}

La escuela de gestion participativa esta acrecentando su influencia sobre las empresas, particularmente en el seno de las PyME.

Este renuevo se funda en dos elementos:

- el creciente interés sobre la funcion GRH (Gestion de Recursos Humanos) en las organizaciones;

- la emergencia de una familia de tecnicas de gestion innovadoras que se basan en el llamado a la responsabilidad colectiva y a la iniciativa de los actores.

El ase logra mas junios que cada uno por su ladow expresa la idea de que el todo puede ser superlor a la suma de las partes en el campo de la accion. 
Este articulo presenta una encuesia hecha en PyME francesas, en las cuales in problematica y lo que esta en juego es la situacion de las formas de gestion y de regulacion inferna de la toma de decision. La encuesta reposa en el conocimiento del funcionamiento real de las estructuras y dispositivos participativos.

Le eleccion de las PyME se basa en la constatacion siguiente : les métodos de administracion en ellas son diferentes de aquellas de las grandes empresas $y$, por consecuencia, las PME constituyen un campo de investigacion propio.

La exposicion de los resultados de esla investigacion se realiza en tres partes.

La presentacion de la filiacion teorica de la gestion participativa considerada como une escuela de gestion de recurses humanos, cuyo eje es el proceso de decision en la empresa. Las referencias a trabajos de gestion y de sociologia de las organizaciones permitent obtener una perspectiva estratégica para la participacion.

A partir de esto, el articulo presenta la construccion de las hipotesis de la investigacion, la encuesta misma y la metodologia de colecta de la informacion.

Finalmento, la tercera parte expone las variedades de dispositivos participativos que se desprenden de la muestra de empresas que se ha utilizado. Dos puntos han sido abordados : su description y los elementos caracteristicos de su funcionamiento. 


\section{Introduction}

Le développement de la participation dans l'entreprise est devenue ces dernières années une idée en vogue développée par l'école dite du management participatif. Ce mouvement plonge cependant ses racines dans une tradition déjà ancienne. Le concept de structures participatives de décision qui en est issu a luimême connu des antécédents en France dès les années 30, avec notamment les travaux de $\mathrm{H}$. Dubreuil. L'effet de mode qui joue actuellement en faveur de la participation ne risque-t-il pas cependant de masquer la variété des conceptions qui lui sont sous-jacentes?

La mise en perspective des principales branches auxquelles l'association de l'entreprise et de la participation a donné naissance sera abordée dans un premier temps. Ceci permettra de définir les hypothèses de travail et les variables étudiées.

Puis, le positionnement particulier de ce travail auprès d'un échantillon de PME sera exposé, afin de restituer le cadre de l'enquête et les conditions de la collecte de l'information. Une synthèse de quelques résultats importants qui, contrairement à de nombreux travaux en ce domaine ne portent pas sur une monographie, sera présentée.

\section{De la filiation du management participatif à des hypothèses de travail}

\subsection{Rappels sur les origines et les tendances des travaux}

L'idée de participation à laquelle se réfère le management participatif s'applique au domaine de la gestion et de la prise de décision. La participation vue sous l'angle de l'actionnariat salarié ou de l'intéressement financier aux résultats est en dehors de son champ de préoccupation, que celui-ci prenne la forme directe de l'entrée du personnel de l'entreprise dans le capital ou soit limité à sa présence au conseil d'administration.

Les tenants de l'école des relations humaines sont les premiers à avoir formalisé et institué le concept de participation, alors compris comme un moyen efficace d'abaisser les résistances aux changements des groupes ou des individus (Bernoux, 1985). Mais, comme le souligne fort opportunément cet auteur, la vision qui a prévalu était de l'ordre de la psychologie behavioriste, lequel n'envisage que le couple stimuli-réponse à l'exclusion de toute autre variable. Ainsi, R. Likert a pu montrer dès les années 40 la supériorité du système 4 d'organisation des entreprises, le système participatif qui, selon lui, est de loin le plus efficace et correspond de surcroît à la préférence des dirigeants.

A un autre niveau hiérarchique de l'organisation et avec, par conséquent, des groupes de travail différents, le management par objectif (la DPO-direction par 
objectif), ou encore plus explicitement la DPPO, qui insiste sur le côté participatif (le deuxième P-participative), ont mis en évidence le partage d'objectifs communs entre l'organisation et ses membres, pris isolément ou en tant que groupe formel. Ce type d'approche de la participation est en accord avec un point de vue élargi abordé quelques années au préalable dans les travaux de C. Argyris; ce point de vue s'inscrit également dans le mouvement qui a contribué à la constitution d'une théorie des organisations. Cet auteur a recherché quels étaient les critères d'efficacité d'une organisation (recherche expérimentale, puisqu'il était à la fois universitaire et consultant), insiste cependant sur l'importance des modifications de structure incluant la participation dans le processus de décision comme facteur de succès.

L'apport fondamental de la théorie des organisations est d'avoir démontré que la seule association aux décisions, l'explication du «pourquoi et du comment» de l'action à mener est insuffisante. Son concept de rationalité, appliquéà l'individu, aux groupes et à l'entreprise et son complément inséparable, la perspective stratégique, permettent de comprendre pour quelles raisons «Participer c'est s'engager, et tout le monde ne souhaite pas le faire», comme le rappelle R. Bernoux (1985).

Sur la base de travaux récents, R. Sainsaulieu (1987) situe lui aussi clairement la participation dans une perspective stratégique, indissociable des démarches visant à l'expression et à la démocratie dans l'organisation. Les programmes de Démocratie Industrielle et l'expérience des Kibboutz figurent pour cet auteur comme des références obligées. Son approche exclusivement sociologique est cependant limitée à l'étude du fonctionnement des organisations et des groupes de travail qui les composent. L'efficacité et la performance, incluses dans le modèle de C. Argyris derrière la notion du succès, sont absentes du modèle de l'analyse stratégique, tel qu'il découle des travaux de M. Crozier et du mouvement qui s'y rattache.

Ainsi, un champ de recherche se dégage d'un ensemble de travaux. Construit par opposition aux mouvements tayloriens et bureaucratiques (Sainsaulieu, 1987), il s'écarte également du modèle des relations humaines, qui lie la participation à l'intégration individuelle. Ce champ de recherche pourrait se définir comme l'ensemble des démarches, des expériences et des efforts visant à donner un contenu concret et stratégique à la participation conçue comme une véritable influence du personnel,et des groupes d'acteurs sur les décisions de l'organisation au sein d'un système de négociation permanente (Martin, 1989). Il est par ailleurs intéressant de souligner avec R. Sainsaulieu (1987), que les projets participatifs ne rejettent pas l'organisation syndicale, mais l'obligent à passer du niveau revendicatif/défensif, à l'inventif/propositionnel.

\subsection{Vers des hypothèses de travail pour une enquête}

Rares sont les travaux ayant servi de support expérimental ou pragmatique aux auteurs abordant le champ de la participation aux décisions dans l'organisation 
et qui se soient déroulés au sein de moyennes ou de grandes organisations. Cela est vrai pour les auteurs nord-américains mentionnés ci-dessus qui relatent exclusivement des actions de recherche et/ou de conseil dans des grandes firmes, même si parfois il s'est agit d'une filiale ou d'une unité de production de taille moyenne. Mais il en va de même pour les exemples donnés par R. Sainsaulieu lorsqu'il analyse des exemples français de nouvelle culture d'entreprise, dont la participation est une des composantes. Les situations où l'on rencontre des PME sont extrêmes : l'organisation est en crise, sa survie est en jeu, et le dirigeant fait alors appel à tout le personnel pour se mobiliser en un dernier effort pathétique afin de sauver ce qui peut encore l'être. La proposition n'est pas alors de remettre en cause un fonctionnement de l'organisation en période d'activité normale, mais de rassembler toutes les énergies disponibles pour surmonter une situation de crise aiguë (d'après le sens donné par $B$. Bettelheim à ce concept forgé pour analyser des circonstances où l'individu est menacé de perdre son intégrité).

La littérature sociologique et managériale mentionne de nombreux comptes-rendus monographiques d'entreprises ayant mené des démarches participatives. Plusieurs auteurs (Mothé-Gautrat, Martin, Kissler, Retour, Brus et Obrajovic) se sont intéressés à la mesure de l'intensité de la participation à partir de méthodes largement inspirées de la sociologie quantitative. Un mouvement IDE «Industrial Democracy in Europe» est parvenu à regrouper pour un temps la plupart de ces chercheurs autour d'un projet d'approches comparatives menées simultanément, fait de rencontres et de publications communes.

Les évaluations de nature plus qualitative s'intéressent également en priorité à des expériences vécues dans des grandes organisations (se référer à la liste de cas cités par R. Sainsaulieu dans sa «Sociologie de l'organisation de l'entreprise»). Et encore, faudrait-il établir finement la distinction entre une démarche participative sur un projet ponctuel, ainsi que la mise en place d'un dispositif participatif (Martin, 1989) à caractère permanent qui soit en soi un élément stratégique de la gestion de l'organisation. Ces deux cas de figure se rejoignent cependant sur une conception commune de la GRH qui n'est plus perçue de manière réductible comme une gestion de moyens, mais s'insère dans une culture participative, associée à des stratégies et des projets de développement (Sainsaulieu).

Par opposition ou complémentarité à ces approches avant tout sociologiques, l'approche socio-économique (H. Savall) est, dans ses fondements, d'inspiration gestionnaire. En effet, ici c'est moins une méthode d'analyse et d'évaluation du fonctionnement des organisations qui est proposée qu'un mode d'intervention organisée rattaché au courant de la recherche-action. S'il est incontestable que l'on y retrouve les notions clés d'objectifs et de négociation (éléments clés d'un dispositif participatif), cette approche prend appui sur une «technologie» de l'intervention comparable à celles mises en oeuvre par les grands cabinets de conseil en organisation. La marge de créativé et d'initiative des acteurs est contrainte par le dispositif de l'intervention, et la négociation est asservic au but défini pour une intervention socio- 
économique. Se pose alors la question de la finalité de cette démarche : ne serait-elle pas plus motivationnelle que participative?

Partant de ce constat d'ensemble, les hypothèses de base à cette enquête s'inscrivent dans le cadre décrit ci-après.

D'une manière générale, sur la masse des travaux publiés, un faible pourcentage de recherches en gestion portent sur les PME. Parmi celles-ci, on peut retrouver plus particulièrement cinq thèmes : le financement, l'exportation, la RD et les transferts de technologie, les relations avec les grandes entreprises dans la position de sous-traitant, et enfin, la fonction de dirigeant dans une structure de type familial. Ainsi, à l'insuffisance quantitative, s'ajoute une absence presque totale de recherches sur la GRH. Avant les travaux de H. Mahé, il n'existait pas de contribution dressant un panorama de la fonction GRH dans la PME.

La problématique et les enjeux de cette recherche reposent également sur le fait que les entreprises sont actuellement confrontées au besoin de faire évoluer leurs modalités de gestion et leur mode de régulation interne. Les efforts déployés au sein des entreprises pour accroître les cercles de qualité, les projets d'entreprise et le concept de qualité totale comme facteur structurant de l'action de l'organisation nous ont incité à décrire et évaluer le fonctionnement réel des éléments liés aux structures participatives influençant le personnel dans les PME. Les PME ont été retenues pour les raisons évoquées précédemment d'une part, mais aussi parce que les méthodes managériales de direction y sont différentes de celles pratiquées par les grandes entreprises. Les PME constituent par conséquent un champ d'investigation en soi.

Sans prétendre reprendre un point de vue exhaustif de la fonction «personnel» dans la PME, cette enquête a adopté un point de vue central de l'entreprise, qui constitue le cadre de la réflexion. Il implique une conception stratégique globale de la GRH.La fonction GRH de l'entreprise n'est plus appréhendée isolément au travers de la gestion du personnel perçue comme un moyen, mais véritablement comme une modalité de gestion. En d'autres termes, la participation aux décisions et le dispositif participatif, (ce que nous avons intitulé par ailleurs le participationnel), sont plus qu'une méthode de direction de l'entreprise. C'est à l'idée de conception globale des structures de l'organisation qu'on fait référence ici. Le cadre de l'enquête est conçu pour identifier le projet stratégique de gestion de l'entreprise, qu'il ait été ou non explicité.

Il est vite apparu clairement qu'il ne serait pas possible de s'adresser aux entreprises retenues dans l'échantillon en leur parlant d'emblée d'un dispositif qui dans la plupart des situations est inexistant. A l'opposé, un questionnaire détaillé qui vise à faire ressortir des aspects essentiels du mode de fonctionnement réel de l'organisation a pu être présenté sans difficulté majeure. 


\section{L'enquête et la méthode de collecte de l'information}

Sans entrer dans les détails de la méthodologie de cette enquête, il paraît néanmoins important de décrire les conditions de son déroulement, lesquelles sont inhérentes à la conception du questionnaire.

\subsection{La méthode de collecte de l'information}

Les entreprises faisant partie de l'effectif des enquêtes sont elles-mêmes issues d'un échantillon non représentatif des PME françaises. Lorsque la question relative à l'échantillonnage s'est posée, elle s'est d'emblée révélée inséparable de l'obtention d'un rendez-vous aux fins d'une interview relativement longue ( $1 \mathrm{h.30}$ à $2 \mathrm{~h}$. par entretien). Le fait de savoir s'il pouvait être possible d'obtenir une recommandation facilitant les démarches a donc pesé sur la méthode.

L'idéal d'un échantillon totalement aléatoire de PME a été rapidement exclu pour deux raisons : la première repose sur l'hypothèse subjective qu'il est peu probable que sur un tel sujet, une cinquantaine de réponses «au hasard» permettent de rencontrer plus d'une ou deux expériences originales ou intéressantes. De plus, les entreprises, qui par cette méthode auraient accepté de répondre à un questionnaire comprenant un vaste ensemble de questions détaillées relatives à leur fonctionnement, se seraient déjà sélectionnées d'elles-mêmes et auraient constitué par conséquent un échantillon nettement plus restreint.

La seconde raison de ce choix a concerné le coût de l'opération. Ce coût a déjà été élevé en soi avec la méthode retenue. L'échantillon aléatoire serait revenu encore plus cher, sans garantir pour autant des résultats plus intéressants que ceux obtenus.

L'option retenue a donc consisté à utiliser un interlocuteur privilégié d'une association de dirigeants d'entreprises, qui apporte soutien et recommandation pour les prises de contact. Ainsi, les résultats de l'enquête sont à interpréter en tenant compte d'une information supplémentaire importante; toutes les entreprises ayant répondu ont leur dirigeant affilié à une association dont les membres partagent une philosophie commune du management du personnel. Elle se manifeste en particulier par la recommandation de l'adoption d'une structure de gestion originale située entre un $\mathrm{CE}$ avec une attribution économique étendue (organisme légal), un comité de direction élargi, et un organe de consultation médiatisé de tout le personnel. En tout état de cause, cette recommandation incite à développer la participation du personnel aux décisions, et, dans la charte de l'association, ce thème est explicitement repris.

L'enquête porte donc sur des PME qui, par le fait de l'appartenance de leur dirigeant à cette association, devraient toutes présenter des formules plus ou moins développées de structures participatives. On devait par conséquent pouvoir apprécier d'une part comment se traduit pratiquement une recommandation librement adoptée 
par les dirigeants des entreprises en question, et d'autre part obtenir un échantillon intéressant de modes de diffusion du concept de participation dans les PME a priori favorables.

A la différence des approches sociologiques du champ de la participation, les entretiens n'ont pas porté sur les opinions, comportements, avis et attitudes des acteurs de l'organisation seuls ou en groupe, mais sur l'entreprise comprise comme une entité en soi.

Cela veut dire que l'interlocuteur chargé de répondre devait faire l'effort de réfléchir à ce qui se passe, ce qui se vit dans l'organisation au quotidien, en s'abstrayant de toute opinion personnelle. Il est évident qu'il est illusoire de prétendre exclure toute subjectivité, mais les questions ont été conçues de telle sorte que plusieurs acteurs de l'organisation soient en mesure d'y répondre au nom de l'entreprise, et que ces réponses multiples, si elles avaient existé, auraient dû être semblables à l'intérieur d'une même firme.

Aucun questionnaire n'est passé deux fois dans la même organisation, et aucun questionnaire n'a pu être rempli collectivement par plusieurs personnes, ce qui aurait amélioré notablement l'objectivisation recherchée. En effet, si pour chaque entreprise de l'échantillon il avait été possible de réunir 3 ou 4 acteurs chargés de donner une réponse unique à chaque question, les sensibilités personnelles dont il est impossible de se départir totalement auraient pualors être considérablement réduites.

Une condition sine qua non posée lors de la prise de contact était que la personne chargée de répondre soit de préférence le dirigeant en titre de l'entreprise. Lorsque dans quelques cas cela n'a pas été possible, l'exigence «minimale» était que l'interlocuteur possède une vision d'ensemble de l'organisation. L'adjoint du dirigeant, lorsque ce poste existait, pouvait répondre valablement aux questions. Il est certain que pour les petites entreprises, seul le dirigeant possède une vision complète de l'organisation. C'est pour cette raison par exemple que plusieurs propositions de rencontre avec «le comptable qui connaît tout aussi bien que moi» ont été écartées. Quand on connaît les agendas de bon nombre de patrons de PME, les difficultés à obtenir des rendez-vous s'imaginent sans peine.

\subsection{La sélection des enquêtes et le questionnaire}

Le caractère familial de beaucoup de PME a impliqué que cette enquête comporte une sélection rigoureuse des entreprises retenues. De plus, pour éviter le travers de l'uniformité de procédures et de modalités de gestion qui se rencontrent parfois au sein des groupes, plusieurs critères de sélection ont été mis en avant.

Afin d'éviter de faire figurer des organisations imprégnées d'un mode de gestion strictement familial, la limite inférieure de taille a été fixée à un effectif de 30 salariés. Il a en effet été retenu comme hypothèse, que ce seuil constituait une limite plausible pour que la firme éprouve la nécessité d'une «organisation» et d'une structure. La limite supérieure a été arbitrairement fixée à 500 salariés, ce qui a donné un panel assez large d'entreprises. 
La non appartenance à un groupe, même si ce dernier a pour principe d'accorder une large autonomie à ses filiales, a constitué le second critère retenu pour figurer parmi la population à laquelle s'adresse le questionnaire. Cette condition d'indépendance repose sur le fait qu'il s'est avéré souhaitable que le dirigeant d'entreprise dispose réellement de la possibilité de mettre en oeuvre une conception de la GRH en toute indépendance, et sans avoir à en référer à une autorité de tutelle.

Les entreprises de sous-traitance dont l'activité principale et le fonctionnement sont comparables en de nombreux points à ceux d'un atelier de fabrication ont été exclues. Un critère de sélection sévère a consisté à ne pas inclure une entreprise qui ne s'adresse pasà un marché, parce qu'en situation dominante de dépendance visà-vis d'un client.

Ensuite, c'est en fonction de l'implantation de l'association que les interviews se sont déroulées. L'Ile-de-France est sous-représentée au regard de la province, mais ce point n'est-il pas aussi le reflet de la répartition géographique des PME sur le territoire? La proportion d'entreprises industrielles est sensiblement plus élevée que celle des services dans l'échantillon. Aucune entreprise atypique ne figure dans l'effectif. Il s'agit par exemple de cas où le personnel n'est pas rassemblé en un même lieu de travail (transport, entretien-dépannage, entreprises de travail temporaire,...), et qui éprouve de ce fait des besoins de structuration de nature différente.

Le questionnaire par lui-même est long, puisqu'il nécessite un entretien dont la durée totale varie de $1 \mathrm{~h} .30$ à $2 \mathrm{~h}$., pour être sûr de le remplir complètement. Au total, ce sont 44 questionnaires complets qui ont donné lieu à une exploitation statistique.

Sur le champ pris en considération, la technique de l'enquête présente des limites évidentes. Quand il s'agit d'apprécier la politique de GRH et le degré de participation du personnel, l'utilisation d'un questionnaire, dont presque exclusivement toutes les questions sont fermées, peut donner lieu à des interrogations. Des discussions préalables avec des tenants de l'analyse stratégique des organisations ont permis de mesurer quelques unes des réticences à l'égard de ce type d'approche.

Plusieurs arguments ont néanmoins conduit à poursuivre dans la voie développée ici.

En premier lieu, il est clair qu'une approche stratégique, qui est par essence qualitative et représente une intervention lourde dans chaque entreprise, ne saurait s'appliquer d'emblée à 44 entreprises. De surcroît, ces analyses sont sociologiques par nature, et ont comme finalité première de décrire et d'expliquer des phénomènes, non d'avancer des propositions d'action. Or, même s'il arrive souvent que les travaux de science de gestion ne soient pas toujours en phase avec l'attente des organisations en matière d'aide à la décision et de recommandation, ils tentent le plus souvent d'intégrer cette préoccupation dans la définition de leur sujet de recherche.

Il a ensuite paru intéressant de retenir comme une des hypothèses de la recherche, qu'un questionnaire qui consacre un temps très court par entreprise en comparaison avec une approche qualitative de longue haleine du type de celle que 
nous avions déjà menée une première fois puisse, malgré tout, produire des résultats intéressants.

Il est sûr toutefois que l'image de la marche réelle de chaque entreprise n'aura été consignée que partiellement lors de chaque entrevue; l'essentiel étant ici de mesurer les limites de la démarche, et de ne pas chercher à faire dire aux états statistiques plus de choses qu'il est raisonnable d'en attendre. La qualité standard de l'information collectée devait être garantie par le fait que tous les questionnaires ont été remplis par une seule personne,à laquelle, de surcroît, on présentait dès le début de l'entretien les buts et l'esprit de l'enquête.

\section{Des dispositifs participatifs variés, combinés à des fonctionnements différenciés}

Trois aspects de cette enquête seront abordés ici sous une forme résumée. Au préalable, la composition de la population d'entreprises soumise au questionnaire sera précisée.

Ce sont 44 PME, PMI en majorité, qui ont principalement à leur tête des propriétaires dirigeants masculins. Seules 6 firmes ont une femme comme dirigeante, ce qui fait une moyenne de $7 \%$ alors que les femmes occupent en moyenne, $17 \%$ des postes cadres.

L'effectif salarié est parfois complété par du personnel sur contrat à durée déterminée mais dans une proportion inférieure à 5\% de l'effectif dans $80 \%$ des cas.

L'échantillon comprend 36 sociétés anonymes, 6 SARL et 2 SCOP (dans le secteur du bâtiment). La structure de direction induite pour les SA et les SCOP ne présente qu'un seul cas avec directoire et conseil de surveillance.

Le profil d'ensemble de l'échantillon révèle des entreprises relativement stables et classiques; pas de création récente (moins de 5 années d'existence), peu de modifications de grande ampleur (l'évolution des effectifs est très rarement supérieure à une variation de $10 \%$ en 2 ans), et pour la majorité, une situation économique et financière qui ne fait pas peser de menaces sur la survie à court terme de l'entreprise.

\subsection{Les dispositifs participatifs}

Une représentation des structures formelles de participation et d'influence du personnel sur les décisions est présentée dans le tableau ci-après.

Toutes les entreprises ont mis en place au moins l'une ou l'autre des structures mentionnées. Il est évident que le degré d'implication des acteurs dans le processus de décision ne connaîtra pas la même intensité selon la nature de la ou des structures dont il fait partie. Les deux types qui prédominent, à savoir la réunion 
fonctionnelle de service et l'assemblée de tout le personnel n'impliquent pas les mêmes observations. La première montre qu'elle correspond à un mode de gestion apparemment répandu, alors que pour l'assemblée du personnel, c'est, semble-t-il, une des options défendues par le groupement qui est bien suivie, puisque les trois quarts d'adhérents pris au hasard ont adopté cette pratique.

On oberve donc deux attitudes : l'une proche du personnel d'exécution que l'on pourrait qualifier de méfiante à l'égard des structures innovatrices et qui ne se retrouve qu'au sein d'une minorité d'entreprises de l'échantillon. L'autre serait volontariste quand il s'agit d'instaurer un type de réunions qui aurait pu être largement expérimenté, et qui, pour les assemblées annuelles de personnel, est chargée symboliquement.

Tab. 1

\begin{tabular}{|l|c|c|}
\hline Structures formelles de décision & OUI & NON \\
\hline Comité de direction & 17 & 27 \\
\hline Réunion fonctionnelle de service & 38 & 6 \\
\hline Cercle de qualité ou de progrès & 10 & 34 \\
\hline Groupe d'expression directe & 12 & 32 \\
\hline Réunion d'information du personnel & 33 & 11 \\
\hline Assemblée d'atelier, département & 15 & 29 \\
\hline
\end{tabular}

(Le total de chaque ligne est égal à l'effectif enquêté, soit 44.)

\subsection{Le fonctionnement du dispositif}

Si on essaie d'entrer plus en détail dans le fonctionnement du dispositif participatif, il faut nécessairement décrire au moins une des structures en présence. En demandant à chaque personne interviewée de choisir une structure décisionnelle de l'entreprise pour en étudier les rouages, on s'assure d'étudier une partie du dispositif qui importe dans le vécu quotidien des acteurs de l'entreprise. Parmi toutes celles qui ont été citées, un regroupement dégage deux types dominants : les réunions fonctionnelles, mentionnées 14 fois (32\%), et les comités de direction, 18 fois (41\%).

Dans un tiers des cas, la fréquence de réunion est hebdomadaire, et dans un quart des autres cas, elle est mensuelle. On relève une grande stabilité des participants, qui sont à peu près toujours les mêmes personnes. L'éventail hiérarchique des membres de ces structures est plus étendu pour les réunions fonctionnelles. Pour l'ensemble, tous types confondus, dans 21 cas des ouvriers (19) ou des employés (2) 
représentent le niveau de qualification le plus faible, et 37 réponses sur le total indiquent que le chef d'entreprise assiste aux réunions. Cette forme d'implication dü dirigeant est très révélatrice de la façon dont marchent les PME.

Le tableau 2 fournit une mesure du degré de formalisme des réunions.

Tab. 2

\begin{tabular}{|l|c|c|}
\hline Degré de formalisme & OUI & NON \\
\hline Ordre du jour & 36 & 6 \\
\hline Compte-rendu de réunion & 21 & 21 \\
\hline Relevé de décisions & 30 & 11 \\
\hline Durée fixée de réunion à priori & 29 & 11 \\
\hline Durée fixée selon les besoins & 10 & 30 \\
\hline Durée de réunion variable & 6 & 35 \\
\hline Présidence/animation hiérarchique & 33 & 9 \\
\hline
\end{tabular}

(Le total des réponses à ces questions est de 42.)

Ces réunions sont relativement structurées, puisque dans une très grande majorité de cas, un ordre du jour est préparé. La durée est prévue à priori, et la pratique du relevé de décisions est généralisée dans $75 \%$ des situations. Les réponses sont en revanche partagées sur la question de la rédaction régulière d'un compte-rendu de réunion. Cette pratique crô̂t assez rapidement avec la taille de l'entreprise.

La durée moyenne de chaque réunion excède rarement 4 heures $(2$ cas sur le total des réponses).

En résumé, ce sont des réunions préparées, où se retrouvent régulièrement des personnes appelées à travailler ensemble selon un schéma partagé par tous.

Les objectifs et le rôle assignés à ces structures sont repris dans le tableau 3 (p. 102).

Ce tableau révèle essentiellement que dans trois cas sur quatre, la structure retenue spontanément a pour fonction d'élaborer une solution collective. Ainsi, lorsqu'il n'est plus question d'aborder le thème de la participation au sein de structures dûment référencées, lorsque ce sont des aspects de la vie quotidienne des organisations qui sont observées, les possibilités d'influence du personnel sur les décisions existent. 
Tab. 3

\begin{tabular}{|l|c|c|}
\hline Rôles attribués à la structure type & OUI & NON \\
\hline Information & 34 & 7 \\
\hline Connaître les avis & 33 & 10 \\
\hline Élaborer une solution & 31 & 10 \\
\hline Assurer une équipe & 23 & 17 \\
\hline Autres fonctions & 19 & 22 \\
\hline
\end{tabular}

Ces possibilités sont encore limitées, car les réponses aux questions concernant le type de problèmes abordés par ces structures montrent que la gestion courante (36 cas) et les décisions managériales (33 cas) sont citées dans plus de $80 \%$ des situtations. A la question ayant trait aux décisions touchant l'orientation stratégique, il a été répondu oui 19 fois, et non dans 23 cas.

La démarche suivie lors de l'introduction ou du développement de l'informatique s'apparente-t-elle ou non à une procédure participative? En d'autres termes, une modification importante de l'organisation des tâches, et des postes de travail sera-t-elle un moment privilégié pour accroître la place occupée par l'espace participatif de l'entreprise?

Les réponses fournies sont résumées dans le tableau 4.

Tab. 4

\begin{tabular}{|l|c|c|}
\hline Démarche lors de l'informatisation & OUI & NON \\
\hline Information du personnel & 27 & 7 \\
\hline Information par la hiérarchie & 23 & 11 \\
\hline Concertation avec les cadres & 25 & 8 \\
\hline Concertation avec le personnel & 9 & 26 \\
\hline Création d'une commission ad hoc & 12 & 23 \\
\hline Recours à une SSI & 21 & 14 \\
\hline Installation par le fournisseur & 14 & 20 \\
\hline
\end{tabular}


Ces réponses démontrent aisément les limites imposées à l'exercice de l'influence du personnel sur les décisions. Un investissement informatique est, pour une PME, bien souvent le fruit d'une décision stratégique. Or, les réponses précédentes avaient déjà révélé que pour le dirigeant de PME, dans 3 cas sur 4 , les décisions stratégiques font partie du domaine réservé. Dès lors; le fait que la concertation du personnel en ce domaine soit peu développée, et que l'instauration d'une commission ad hoc ne le soit guère plus, n'étonne plus.

C'est une des limites au développement du participatif dans les PME, que les plus dynamiques d'entre-elles devraient parvenir à dépasser. Cela a été le cas pour près d'un quart des entreprises de l'échantillon.

\section{Conclusion}

Pour conclure provisoirement, cette enquête aura permis de montrer qu'une majorité de PME de l'échantillon est dotée d'un dispositif participatif. Même si d'aucuns peuvent penser qu'il est léger au regard des expériences habituellement relatées par la littérature de science de gestion ou de sociologie des organisations, ces dispositifs ont le mérite d'exister autrement qu'à l'état de prototype ou d'expérience.

Au sein de ces dispositifs, un groupe de salariés influence les décisions de l'entreprise par sa participation à des instances qui se réunissent régulièrement et qui ont une existence formelle dans l'organisation.

Au cours de l'enquête, aucun dispositif «lourd» n'a été trouvé. Ce qui tendrait à s'interroger sur le bien fondé de conclusions hardies rencontrées parfois en matière de participation du personnel aux décisions, et plus généralement de ce qu'il est convenu d'intituler la culture d'entreprise, même si les concepts sont séduisants dans l'absolu.

Le propre des dispositifs révélés par ce travail est d'être pour la plupart parfaitement intégrés, voire en symbiose, avec l'organisation au sein de laquelle ils ont été observés. Ce ne sont pas des objets que l'entreprise aura eu besoin d'adapter, à l'instar des groupes d'expression directe, des cercles de qualité ou des troupes de progrès.

L'observation qui précède fournit un élément partiel de réponse aux enjeux annoncés pour cette recherche; il existe bien des méthodes spécifiques de management dans les PME qui ne soient pas des outils de gestion standardisés et diffusés d'entreprise en entreprise, à l'instar de ce qui peut s'observer dans les grandes organisations. On souligne trop souvent les capacités d'innovation technologique des PME, en oubliant de s'interroger sur leurs capacités réelles à innover dans le domaine du management des ressources humaines, par l'instauration de mode de régulation interne qui leur soit spécifique. 
Les dispositifs observés sont modestes, mais dépassent cependant les espérances concernant une enquête qui s'est déroulée en vraie grandeur sur un sujet délicat. Leur force réside vraisemblablement dans le fait d'avoir réussi à associer dans une proportion importante de cas la presque totalité des échelons hiérarchiques de l'entreprise.

\section{BIBLIOGRAPHIE}

D’Ambroise, G. et Gasse, Y., (1987), «Strategy making in PME», Doc. n 87-116, Université Laval, Québec.

Bemoux, P., (1985), La sociologie des organisations, Paris, Le Seuil.

Borzeix, A. et Linhart, D., (1988), «La participation, un clair obscur», Sociologie du travail, $\mathrm{n}^{\circ} 1$.

Chalvin, D., (1978), L'entreprise négociatrice, Paris, Dunod.

Ganne, B., (1987), «Pour une sociologie des PME», $2^{\circ}$ journée de sociologie du travail, Lille.

Gautrat, J. et Martin, D., (1984), «Cheminement inventif d'une démarche participative», CRESST-GSCI, Sceaux.

Jalbert, P., (1989), Les ressources humaines, atout stratégique, Paris, L'Harmattan.

Julien, P.A. et Marchesnay, M., (1988), La petite entreprise, Paris, Vuibert.

Lorino, P., (1989), L'économiste et le manageur, Paris, La Découverte.

Mahé de Boislandelle, H., (1989), Gestion des ressources humaines dans les PME, Paris, Economica.

Martin, D., (1989), «Crise d'identité et gestion de la modemisation», Revue française de gestion, sept. oct.

Mintzberg, H., (1986), Structure et dynamique des organisations, Paris, Éd., d'Organisation.

Reynaud, J.D., (1988), «Les régulations dans les organisations», Revue française de sociologie, $\mathrm{n}^{\circ} 1$.

Sainsaulieu, R., Tixier, P.E. et Marty, M.O., (1983), La démocratie en organisation, Paris, Librairie des méridiens.

Sainsaulieu, R., (1987), Sociologie de l'organisation de l' entreprise, Paris, Dalloz et PNSP.

Stankiewicz, F., (éd.), (1988), Les stratégies d'entreprises face aux ressources humaines, Paris, Economica. 